\title{
A case report of a 4-year-old child with glucose-6-phosphate dehydrogenase deficiency: An evidence based approach to nutritional management
}

\author{
Alex Pinto ${ }^{1}$, Anita MacDonald ${ }^{2}$, Esmeralda Cleto $^{3}$, Manuela Ferreira Almeida ${ }^{1,4}$, \\ Paula Cristina Ramos ${ }^{1}$, Júlio César Rocha ${ }^{1,5,6}$ \\ ${ }^{1}$ Centro de Genética Médica, Centro Hospitalar do Porto - CHP, Porto, Portugal; ${ }^{2}$ Birmingham Children's Hospital, \\ Birmingham, UK; ${ }^{3}$ Centro Materno Infantil do Norte - CMIN, Centro Hospitalar do Porto - CHP, Porto, Portugal; ${ }^{4}$ Unit \\ for Multidisciplinary Research in Biomedicine, Abel Salazar Institute of Biomedical Sciences, University of Porto-UMIB/ \\ ICBAS/UP, Porto, Portugal; ${ }^{5}$ Faculdade de Ciências da Saúde, Universidade Fernando Pessoa, Portugal; ${ }^{6}$ Center for Health \\ Technology and Services Research (CINTESIS), Portugal \\ E-mail : julio.rocha@chporto.min-saude.pt \\ Received: 17th October 2016, Revised: 23rd November 2016, Accepted: 29th December 2016
}

SUMMARY: Pinto A, MacDonald A, Cleto E, Almeida MF, Ramos PC, Rocha JC. A case report of a 4-year-old child with glucose-6-phosphate dehydrogenase deficiency: An evidence based approach to nutritional management. Turk J Pediatr 2017; 59: 189-192.

The objective was to describe the nutritional management of a 4-year-old child with glucose-6-phosphate dehydrogenase (G6PD) deficiency.

A 4-year-old male child, African descent, born from non-consanguineous parents presented with a clinical history of frequent respiratory infections, usually treated with antibiotics. At 30 months of age, G6PD diagnosis was made after eating one portion $(40-60 \mathrm{~g})$ of fava beans, resulting in severe hemolytic anemia hospitalization for 5 days. Diagnosis was confirmed by G6PD activity measurement. Nutritional counseling was given to avoid dietary oxidative stressors particularly the exclusion of fava beans and accidental ingestion of other similar beans. Dietary intake of high vitamin C containing foods was discouraged and adequate hydration advised.

Nutritional management is crucial in preventing acute stress events in patients with G6PD deficiency.

Key words: exercise, fava beans, glucose-6-phosphate dehydrogenase, hemolytic anemia, oxidative stress.

Glucose-6-phosphate dehydrogenase (G6PD) deficiency, an X-linked hereditary genetic error with different variants, is the most common enzymatic deficiency in the world, affecting 400 million people ${ }^{1,2}$. At the pentose phosphate pathway, G6PD catalyzes the conversion of nicotinamide adenine dinucleotide phosphate (NADP) to its reduced form (NADPH), which protects cells against oxidative damage and helps to preserve the reduced form of glutathione $(\mathrm{GSH})$, responsible for destroying hydrogen peroxide and hydroxyl free radicals. When the pentose phosphate pathway is the only way to generate NADPH in erythrocytes, these cells are more susceptible to oxidative stress $^{1,2}$.
The majority of patients with G6PD deficiency are asymptomatic although there is still a risk of neonatal hyperbilirubinemia ${ }^{1,2}$ and acute hemolysis after contact with oxidative stressors from fava bean ingestion, oxidant drugs, chemotherapy, infection and diabetic ketoacidosis ${ }^{3}$.

The severe hemolytic anemia caused by excessive ingestion of fava bean (Favism) is most frequently associated with the Mediterranean variant and seems to be caused by some toxic constituents of fava beans, such as divicine, isouramil and convicine ${ }^{1,2}$. Therefore, nutritional treatment is mainly based on the avoidance of these oxidative stressors ${ }^{1,2}$, and monitoring clinical symptoms and biochemical data. 
Table I. Clinical Biochemistry of the Patient at Acute Phase and Following Nutritional Management [values in brackets represent normal distribution].

\begin{tabular}{ll}
\hline Parameters & Results at acute phase vs. results following nutritional management \\
\hline Erythrocytes & 2.62 vs. $4.59 \times 10^{6} / \mathrm{mm}^{3}[3.9-5.3]$ \\
Hemoglobin & 7.2 vs. $12.4 \mathrm{~g} / \mathrm{dl}[11.5-13.5]$ \\
Hematocrit & 22.5 vs. $37.6 \%[34.0-40.0]$ \\
Mean corpuscular volume & 85.9 vs. $82 \mathrm{fl}[75-87]$ \\
Mean corpuscular Hb & 27.5 vs. $27.1 \mathrm{pg}[23.0-31.0]$ \\
Mean corpuscular Hb concentration & 32.0 vs. $33.0 \mathrm{~g} / \mathrm{dl}[30.0-36.0]$ \\
Red cell distribution width & 16.3 vs. $12.9 \%[11.5-14.5]$ \\
Platelets & 344 vs. $447 \times 10^{3} / \mathrm{mm}^{3}[150-400]$ \\
Mean platelet volume & 10.7 vs. $7.7 \mathrm{fl}[7.2-11.1]$ \\
Platelets distribution width & 12.7 vs. $50.7 \%[25.0-65.0]$ \\
Reticulocytes percentage & 6.59 vs. $2.96 \%[0.5-2.5]$ \\
Reticulocytes absolute value & 172.7 vs. $135.9 \times 10^{3} / \mathrm{mm}^{3}[50.0-100.0]$ \\
Total bilirubin & 7.02 vs. $0.68 \mathrm{mg} / \mathrm{dl}[0.20-1.00]$ \\
Indirect bilirubin & 6.63 vs. $0.43 \mathrm{mg} / \mathrm{dl}[0.00-1.00]$ \\
Lactate dehydrogenase & 663 vs. $263 \mathrm{U} / \mathrm{L} \mathrm{a} 37^{\circ}[120-300]$ \\
Plateletcrit & 0.40 vs. $0.35 \%[0.12-0.36]$ \\
\hline
\end{tabular}

Hb: hemoglobin

Table II. Anthropometric Measurements of the Patient During the Follow-Up.

\begin{tabular}{llllll} 
& \multicolumn{5}{c}{ Time of appointments } \\
\cline { 2 - 6 } Measurements & $32 \mathrm{mo}$. & 36 mo. & 40 mo. & 44 mo. & 53 mo. \\
\hline Weight $(\mathrm{kg})$ & 15.4 & 15.5 & 16.0 & 16.5 & 17.6 \\
Weight SDS & 1.11 & 0.83 & 0.62 & 0.48 & 0.19 \\
Height $(\mathrm{cm})$ & 93.0 & 96.5 & 98.9 & 101.3 & 107.0 \\
Height SDS & -0.17 & 0.34 & 0.19 & 0.15 & 0.20 \\
BMI $\left(\mathrm{kg} / \mathrm{m}^{2}\right)$ & 18.0 & 16.6 & 16.4 & 16.1 & 15.4 \\
BMI for age SDS & 1.73 & 0.90 & 0.73 & 0.56 & 0.08 \\
\hline
\end{tabular}

\section{Case Report}

We present a case report of a 4-year-old child with glucose-6-phosphate dehydrogenase (G6PD) deficiency from Centro Hospitalar do Porto, CHP, Porto, Portugal. Written informed consent was given by the child's mother.

A male child, African descent, born at 38 weeks, had normal neonatal anthropometry (body weight $3130 \mathrm{~g}$; length $47 \mathrm{~cm}$; head circumference $34.5 \mathrm{~cm}$ ). Newborn screening for congenital hypothyroidism and 24 inherited metabolic disorders was negative. Neonatal jaundice occurred with a maximum total serum bilirubin of $15.5 \mathrm{mg} / \mathrm{dl}$, which was promptly treated with phototherapy for 3 days.

At 30 months of age, two days after eating one portion (40-60 g) of fava beans, the child presented with vomiting, fever, jaundice and dark red urine. This led to severe hemolytic anemia (Table I), requiring hospitalization. Diagnosis of G6PD was confirmed by G6PD activity measurement $(5.3 \mathrm{U} / \mathrm{g} \mathrm{Hb}$; normal range: $7.0-20.4 \mathrm{U} / \mathrm{g} \mathrm{Hb}$ ). This was performed after correction of severe hemolytic anemia in order to prevent a false negative result, caused by the hemolysis of older erythrocytes (with higher enzyme deficiency) and the increase in young erythrocytes and reticulocytes (with normal or near-normal enzyme activity) ${ }^{1,2}$. 
Table III. Evidence of Nutritional Management, Exercise and Nutrient Supplementation in G6PD Deficiency.

Nutritional management

- Exclusion of fava bean ingestion (or similar beans): Avoid oxidative stressors (possible effect of divicine, isouramil and convicine) ${ }^{1}$.

- Avoid high vitamin C foods: Ascorbic acid may be associated with hemolysis ${ }^{5}$.

- Cross contamination by fava beans: Reported case of acute event due to cross contamination of pumpkin ${ }^{4}$.

\section{Exercise}

- Monitor exercise closely: Although no greater oxidative stress was found performing exhaustive exercise comparing to normal children ${ }^{7}$, there are no data on longterm training and it is considered that this may increase reactive oxygen species production ${ }^{5}$.

Nutrient Supplementation

- Iron and folic acid: Showed benefits for hemolysis when caused by low blood levels but there is no specific research in G6PD deficiency ${ }^{2}$.

- Vitamin E and selenium: Seem to have some effect on chronic hemolysis due to antioxidant function and improvement of red cell half-life but there is no specific research in G6PD deficiency 8,9 .

- Vitamin E: May protect against reduction of G6PD enzyme induced by heavy training ${ }^{10}$.

- L-cysteine: Protection against oxidative damage in G6PD deficiency ${ }^{11}$.

- $\quad \alpha$-lipoic acid: Up-regulation of antioxidant capacity in G6PD deficiency ${ }^{12}$.

Nutritional counseling involved avoiding fava beans and preventing accidental ingestion of other similar beans like bell beans, English dwarf beans, horse beans, pigeon beans, silkworm beans and tick beans. Advice was given to avoid cross-contamination (use different utensils and avoid direct contact with permitted foods when cooking fava or similar beans) as acute crisis episodes have been reported following consumption of pumpkin containing fava beans $\mathrm{DNA}^{4}$. Intake of high vitamin $\mathrm{C}$ containing foods was discouraged because of its possible association with hemolysis ${ }^{5}$. Adequate hydration was also advised. A high carbohydrate snack together with fluid was given before exercise and immediately post exercise. This was given because fatigue during exercise has been reported and oxidative stress appears to be involved with muscular fatigue ${ }^{6}$.

Biochemical data after acute phase recovery remained within normal ranges (Table I). Longitudinal anthropometric measurements showed normal growth progression (Table II).

\section{Discussion}

Standard nutritional counseling enabled this child with G6PD deficiency to remain in good oxidative stress control, with no new acute events. The main principles of dietary treatment are straight forward (Table III) and exercise guidelines should be the same as for healthy children. Exercise has been little studied in G6PD deficiency but it may increase reactive oxygen species production ${ }^{5}$. Nikolaidis et al. $^{7}$ found that patients are able to perform exhaustive exercise without greater oxidative stress compared with controls. However, clinical symptoms should be monitored and training adapted in order to avoid excessive oxidative stress $^{5}$.

Vitamin and mineral supplementation may be important in G6PD deficiency. Iron and folic acid have shown benefits for hemolysis when caused by low levels of these components but there is no specific research in G6PD deficiency ${ }^{2}$. Vitamin E and selenium seem to have some effect in patients with chronic hemolysis due to antioxidant function and improving effect of red cell half-life ${ }^{8,9}$. Moreover, vitamin E may protect against the reduction of G6PD enzyme activity induced by heavy training ${ }^{10}$. There is also some possible interest in L-cysteine, as it increases both antioxidant capacity and production of glutathione, leading to a higher protection against oxidative damage ${ }^{11}$. Georgakouli et al. ${ }^{12}$ reported that $\alpha$-lipoic acid supplementation led 
to an up-regulation of antioxidant capacity and a possible modulation of blood redox status in patients with G6PD.

Although in our patient clinical stabilization was achieved with a standard approach, more research is welcomed to further analyze the potential benefits of nutrient supplementation, in order to optimize nutritional management in G6PD deficient individuals.

\section{REFERENCES}

1. Cappellini MD, Fiorelli G. Glucose-6-phosphate dehydrogenase deficiency. Lancet 2008; 371: 64-74.

2. Frank JE. Diagnosis and management of G6PD deficiency. Am Fam Physician 2005; 72: 1277-1282.

3. Odièvre M-H, Danékova N, Mesples B, et al. Unsuspected glucose-6-phosphate dehydrogenase deficiency presenting as symptomatic methemoglobinemia with severe hemolysis after fava bean ingestion in a 6-yearold boy. Int J Hematol 2011; 93: 664-666.

4. Zuccotti GV, Redaelli F, Gualdi V, et al. Hemolytic crisis in a G6PD-deficient infant after ingestion of pumpkin. Ital J Pediatr 2014; 40: 71 .

5. Laquale KM. Glucose-6-phosphate dehydrogenase (G6PD) deficiency and exercise. Athlet Ther Today 2009; 14: 26-31.
6. Finaud J, Lac G and Filaire E. Oxidative stress: relationship with exercise and training. Sports Med 2006; 36: 327-358.

7. Nikolaidis MG, Jamurtas AZ, Paschalis V, et al. Exerciseinduced oxidative stress in G6PD-deficient individuals. Med Sci Sports Exerc 2006; 38: 1443-1450.

8. Beutler E. G6PD deficiency. Blood 1994; 84: 3613-3636.

9. Hafez M, Amar ES, Zedan M, et al. Improved erythrocyte survival with combined vitamin $\mathrm{E}$ and selenium therapy in children with glucose-6-phosphate dehydrogenase deficiency and mild chronic hemolysis. J Pediatr 1986; 108: 558-561.

10. Tsakiris S, Reclos GJ, Parthimos T, Tsakiris T, Parthimos $\mathrm{N}$, Schulpis KH. $\alpha$-tocopherol supplementation restores the reduction of erythrocyte glucose-6-phosphate dehydrogenase activity induced by forced training. Pharmacol Res 2006; 54: 373-379.

11. Schulpis KH, Reclos GJ, Parthimos T, Parthimos N, Gavriilidis A, Tsakiris S. L-cysteine supplementation protects the erythrocyte glucose-6-phosphate dehydrogenase activity from reduction induced by forced training. Clin Biochem 2006; 39: 1002-1006.

12. Georgakouli K, Deli CK, Zalavras A, et al. $\alpha$-lipoic acid supplementation up-regulates antioxidant capacity in adults with G6PD deficiency. Food Chem Toxicol 2013; 61: 69-73. 